\title{
Correction to: The Present and Future of Liquid Biopsies in Non-Small Cell Lung Cancer: Combining Four Biosources for Diagnosis, Prognosis, Prediction, and Disease Monitoring
}

\author{
Jillian Wilhelmina Paulina Bracht ${ }^{1,2} \cdot$ Clara Mayo-de-las-Casas $^{1} \cdot$ Jordi Berenguer $^{1} \cdot$ Niki Karachaliou $^{1,3}$. \\ Rafael Rosell ${ }^{1,4,5,6}$
}

Published online: 3 May 2020

(C) Springer Science+Business Media, LLC, part of Springer Nature 2020

\section{Correction to: Current Oncology Reports (2018) 20: 70} https://doi.org/10.1007/s11912-018-0720-z

The original version of this review article unfortunately contained mistakes in the Affiliation and Funding sections. The details are given below:

1. "Universitat Autónoma de Barcelona (UAB), Barcelona, Spain" should be added under Jillian Wilhelmina Paulina Bracht.

2. The correct Funding statement is presented below:

Funding This project has received funding from the European Union's Horizon 2020 research and innovation programme under the Marie Skłodowska-Curie grant agreement No 765492. Work in Dr. Rosell's laboratory is partially supported by a grant from La Caixa Foundation and an Instituto de Salud Carlos III grant (RESPONSE, PIE16/ 00011).
Publisher's Note Springer Nature remains neutral with regard to jurisdictional claims in published maps and institutional affiliations.
The online version of the original article can be found at https://doi.org/ 10.1007/s11912-018-0720-z

Niki Karachaliou

nkarachaliou@oncorosell.com

Rafael Rosell

rrosell@iconcologia.net

Jillian Wilhelmina Paulina Bracht jill94bracht@gmail.com

Clara Mayo-de-las-Casas cmayo@panoncology.com

Jordi Berenguer

jberenguer@panoncology.com
1 Pangaea Oncology, Laboratory of Molecular Biology, Quirón-Dexeus University Institute, Sabino Arana 5-19, 08028 Barcelona, Spain

2 Universitat Autónoma de Barcelona (UAB), Barcelona, Spain

3 Instituto Oncológico Dr Rosell (IOR), University Hospital Sagrat Cor, QuironSalud Group, Viladomat 288, 08029 Barcelona, Spain

4 Institut Català d'Oncologia, Hospital Germans Trias i Pujol, Carretera de Canyet, s/n, 08916 Badalona, Barcelona, Spain

5 Institut d'Investigació en Ciències Germans Trias i Pujol, Camí de les Escoles, s/n, 08916 Badalona, Barcelona, Spain

6 Instituto Oncológico Dr Rosell (IOR), Quirón-Dexeus University Institute, Sabino Arana 5-19, 08028 Barcelona, Spain 